\title{
基于改进自适应遗传算法和二维最大熵的 超声红外热像缺陷识别
}

唐长明 ${ }^{1}$, 钟剑锋 ${ }^{1}$, 钟舜聪 ${ }^{1,2}$, 陈 曼 ${ }^{1}$, 伏喜斌 ${ }^{3}$, 黄学斌 ${ }^{3}$

(1. 福州大学 机械工程及自动化学院光学/太赫兹及无损检测实验室, 福建 福州 350108;

2. 上海大学 机电工程及自动化学院, 上海 200444; 3. 厦门市特种设备检验检测院, 福建 厦门 361000）

摘要: 根据超声红外热像检测图像的特点, 为实现图像中缺陷的识别, 提出了一种结合改进的自适应 遗传算法和二维最大摘的分割方法, 以实现准确、快速地分割出目标缺陷区域。该方法首先对超声红 外图像进行预处理, 得到了去噪后的图像, 然后通过二维最大熵算法选取阈值将图像分为目标区域和 背景区域, 并结合改进的自适应遗传算法, 提高分割速度。实验结果表明, 该方法可以有效地滤除图 像噪声, 相比于穷举法和基于简单遗传算法的二维最大熵分割, 本算法具有较好的分割速度和分割精 度。

关键词: 超声红外热像法; 自适应; 遗传算法; 二维最大熵

中图分类号：TG219 文献标识码：A 文章编号：1001-8891(2020)08-0801-08

\section{Ultrasound Infrared Thermography Defect Recognition Based on Improved Adaptive Genetic Algorithm with Two-Dimensional Maximum Entropy}

TANG Changming ${ }^{1}$, ZHONG Jianfeng ${ }^{1}$, ZHONG Shuncong ${ }^{1,2}$, CHEN Man ${ }^{1}$, FU Xibin ${ }^{3}$, HUANG Xuebin ${ }^{3}$

(1. Laboratory of Optics, Terahertz and Non-destructive Testing, School of Mechanical Engineering and Automation, Fuzhou University

Fuzhou 350108, China; 2. School of Mechatronic Engineering and Automation, Shanghai University, Shanghai 200444, China;

3. Xiamen Special Equipment Inspection Institute, Xiamen 361000, China)

\begin{abstract}
A segmentation method was developed for combining an improved adaptive genetic algorithm with a two-dimensional maximum entropy algorithm based on the image features of ultrasound infrared thermography detection for accurate and rapid segmentation of the target defect region to recognize defects of defect recognition in images. First, the infrared image was processed to obtain a denoised image. Next, the image was divided into the target and background regions using a two-dimensional maximum entropy algorithm; the segmentation speed was improved by combining it with the improved adaptive genetic algorithm. The experimental results showed that this method can effectively filter image noise. Compared with an exhaustive method and the two-dimensional maximum entropy segmentation based on a simple genetic algorithm, the proposed algorithm has better segmentation speed and accuracy.
\end{abstract}

Key words: ultrasound infrared thermography, adaptive, genetic algorithm, two-dimensional maximum entropy

\section{0 引言}

超声红外技术是一项将超声波技术和红外技术 相结合的新兴无损检测技术，它对结构件的表面裂 纹、近表面裂纹、分层和脱粘等界面类缺陷具有良好
的检测效果。相比于其他激励方式的红外热成像技 术, 超声红外热像法是采用超声波作为激励源, 因其 激励强度大、作用时间短, 从而具有检测速度快, 能 实现大面积检测的优势, 并且无需对检测对象进行表 面预处理 ${ }^{[1-3]}$ 。该检测法是利用超声波在缺陷处的衰减 
生热来检测裂纹, 能够在缺陷处选择性加热, 增强了 检测图像的信噪比, 提高了检测的灵敏度, 适用于形 状结构复杂无规则、大重量级的检测对象 ${ }^{[4-5]}$ 。在实验 过程中由于背景噪声、材料不均匀等因素使得红外热 像仪采集到的热像图存在成分复杂、噪声严重、边缘 模糊等缺点 ${ }^{[6]}$, 易造成对缺陷的误判, 同时为了进一 步将超声红外无损检测推向工程应用, 需要实现缺陷 的自动识别。

鉴于此, 本文提出一种对超声激励作用下的红外 图像实现自动检测的方法, 采用图像差分运算和形态 学开运算对图像进行去噪, 结合改进的自适应遗传算 法和二维最大熵对图像进行阈值分割, 提取缺陷区 域, 并进一步利用连通区域标记实现缺陷位置的提 取。

\section{1 二维最大熵图像分割法}

由于一维最大熵分割方法只考虑自身像素点而 没有考虑邻域像素点的信息, 当图像的对比度低、背 景噪声严重时, 灰度直方图不一定有波峰和波谷, 此 时利用一维最大熵法方法进行阈值分割效果不好, 目 标区域和背景区域不能很好地区分开。为此, 一些学 者提出了二维最大熵阈值分割的方法, 该方法是一种 结合本身像素点灰度信息和邻域灰度值信息的阈值 分割方法 ${ }^{[6-7]}$ 。

假设一张大小为 $M \times N$, 具有 $L$ 级灰度值红外图 像的二维直方图的 xoy 平面图如图 1 所示。图中横坐 标为原图像 $f(i, j)$ 的灰度级, 纵坐标为对原图像经过平 滑处理后求得的图像 $g(i, j)$ 的灰度级, 区域 1、2 代表 的是目标区域和背景区域, 区域 3、4 代表的是噪声 和边界。二维最大熵分割方法是通过选取一定的阈值 $(s, t)$, 使得区域 1 和区域 2 的熵之和为最大值。

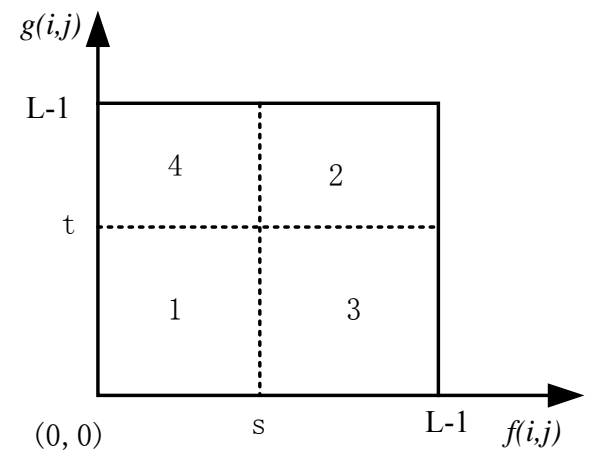

图 1 二维直方图投影图

Fig.1 Two-dimensional histogram projection 图像的二维直方图可由公式(1)计算得出:

$$
P_{x, y}=\frac{h(x, y)}{M \times N}
$$

式中: $h(x, y)$ 为原图像中灰度值为 $x$ 、且邻域平均灰度 值为 $y$ 的像素点的个数; $M$ 和 $N$ 分别为图像的横向、 纵向分辨率。假设分割的阈值选为 $(s, t)$, 则红外图像 中目标区域 1 和背景区域 2 的概率分别为:

$$
\begin{aligned}
& P_{1}=\sum_{x=0}^{s-1} \sum_{y=0}^{t-1} P_{x, y} \\
& P_{2}=\sum_{x=s}^{L-1} \sum_{y=t}^{L-1} P_{x, y}
\end{aligned}
$$

则红外图像中目标区域 1 和背景区域 2 的熵分别 为:

$$
\begin{aligned}
& H_{1}=-\sum_{x=0}^{s-1} \sum_{y=0}^{t-1}\left(P_{x, y} / P_{1}\right) \ln \left(P_{x, y} / P_{1}\right) \\
& H_{2}=-\sum_{x=s}^{L-1} \sum_{y=t}^{L-1}\left(P_{x, y} / P_{2}\right) \ln \left(P_{x, y} / P_{2}\right)
\end{aligned}
$$

将红外图像的二维熵函数 $H(s, t)$ 定义为:

$$
H(s, t)=H_{1}+H_{2}
$$

当熵函数 $H(s, t)$ 取得最大值时, 对应的 $\left(s^{*}, t^{*}\right)$ 即为 二维最大熵阈值:

$$
\left(s^{*} t^{*}\right)=\max _{\substack{0<s<L-1 \\ 0<t<L-1}}[H(s, t)]
$$

\section{2 改进的自适应遗传算法}

遗传算法 (Genetic Algorithms,GA) 是由 Michigan 大学的 J.Holland 教授提出的一种模拟达尔文生物进 化论的寻找全局最优值搜索算法 ${ }^{[8-9]}$ 。遗传算法是将随 机生成几个初始解作为初始群体即第一代, 然后初始 群体像生物进化一样进行 “优胜劣汰” 的选择、交叉 繁殖、基因变异等, 产生生存能力更强、性能更优于 子代群体, 子代群体又作为新父代产生新的子代群 体, 如此循环, 得到的解越来越靠近最优解 ${ }^{[10-11]}$ 。

简单遗传算法存在着一些缺点, 比如收玫性差、 求解速度慢、容易陷入局部最优解。国内外有许多学 者针对简单遗传算法的缺点展开了大量的研究, 提出 了许多不同的改进遗传算法，使遗传算法的性能得到 了很大程度的改善，提高了遗传算法的适用性。本文 在此基础上, 对简单遗传算法中的 3 个遗传算子进行 改进来提高遗传算法的计算速度和收敛性。

本文算法的基本流程: 
1) 编码。本文采用的是二进制编码, 由于图像 的灰度级为 256 , 故每个个体的二进制编码为 16 位, 前面 8 位为原红外图像的阈值 $s$, 后面 8 位为对原红 外图像经过平滑处理后图像的阈值 $t$ 。

2）生成初始群体。本文采取完全随机的方法在 可行解空间生成初始群体。通常种群规模的数量为 $20 \sim 100$, 本文的种群规模设定为 20 。

3 ) 适应度函数。本文采取的适应度函数为红外 图像的二维熵函数。

4) 遗传算子。本文的选择算子同时结合轮盘奢 选择法和最优个体保留。本文算法采取 $10 \%$ 的最优 个体保留的方法, 即每次进行轮盘奢选择前, 先从 20 个群体中选择 2 个最优个体无条件复制进入下一 步操作。

在简单遗传算法中, 交叉率 $P_{\mathrm{c}}$ 与变异率 $P_{\mathrm{m}}$ 均是 采用固定值, 这使得取值较为困难, 若取值太大在算 法后期会破坏优秀个体, 太小则使得算法前期搜索能 力较差 ${ }^{[12]}$ 。针对此问题, SRINVIVAS 等人曾提出了 一种交叉率 $P_{\mathrm{c}}$ 和变异率 $P_{\mathrm{m}}$ 随着种群进化而自动调整 的自适应基本遗传算法。该算法的交叉率 $P_{\mathrm{c}}$ 和变异率 $P_{\mathrm{m}}$ 的调整如式(8)和式(9)所示 ${ }^{[13]}$ :

$$
\begin{aligned}
& P_{\mathrm{c}}= \begin{cases}\frac{k_{1}\left(f_{\max }-f^{\prime}\right)}{f_{\text {max }}-f_{\text {avg }}} & f^{\prime} \geq f_{\text {avg }} \\
k_{2} & f^{\prime}<f_{\text {avg }}\end{cases} \\
& P_{\mathrm{m}}= \begin{cases}\frac{k_{3}\left(f_{\max }-f\right)}{f_{\text {max }}-f_{\text {avg }}} & f \geq f_{\text {avg }} \\
k_{4} & f<f_{\text {avg }}\end{cases}
\end{aligned}
$$

式中: $k_{1} 、 k_{2} 、 k_{3} 、 k_{4}$ 为常数, 值域为 $(0,1) ; f_{\text {max }}$ 当代 群体中的最大适应度值; $f_{\text {avg }}$ 为当代群体平均适应度 值; $f^{\prime}$ 为当代待交叉染色体对中的较大适应值; $f$ 为待 变异个体的适应度值。

从上述两个公式可以看出, 该算法的交叉率 $P_{\mathrm{c}}$ 和变异率 $P_{\mathrm{m}}$ 随着种群的进化而变小, 可以提高收玫 速度。但是, 该算法存在着不足, 当待交叉个体或者 待变异个体的适应度值很接近或者等于群体中最大 适应度值时, 交叉率 $P_{\mathrm{c}}$ 和变异率 $P_{\mathrm{m}}$ 直接等于 0 , 这 种情况要是发生在算法早期的局部最优个体上, 会使 算法收玫于具有最优解, 形成伪解。因此, 本文在此 基础上, 采用改进的算子, 即使在算法几乎收玫的时 候, 交叉率 $P_{\mathrm{c}}$ 和变异率 $P_{\mathrm{m}}$ 仍然不为 0 , 该算子如式(10) 和式(11)所示:

$$
\begin{gathered}
P_{\mathrm{c}}= \begin{cases}k_{1} \cdot \mathrm{e}^{\frac{f_{\text {avg }}-f^{\prime}}{f_{\max }-f_{\text {avg }}}} & f^{\prime} \geq f_{\text {avg }} \\
k_{1} & f^{\prime}<f_{\text {avg }}\end{cases} \\
P_{\mathrm{m}}= \begin{cases}k_{2} \cdot \mathrm{e}^{\frac{f_{\text {avg }}-f^{\prime}}{f_{\max }-f_{\text {avg }}}} & f^{\prime} \geq f_{\text {avg }} \\
k_{2} & f^{\prime}<f_{\text {avg }}\end{cases}
\end{gathered}
$$

式中: $k_{1}$ 为最大变异率, 在本算法中将其取为 0.9 ; $k_{2}$ 为最大变异率, 在本算法中将其取为 0.1 。从式中 可以看出, 经过改进的算子在算法的初始阶段交叉率 $P_{\mathrm{c}}$ 和变异率 $P_{\mathrm{m}}$ 都取较大值, 提高算法的搜索性能, 加快搜索速度, 同时在算法的后期阶段, 适当地降低 交叉率 $P_{\mathrm{c}}$ 和变异率 $P_{\mathrm{m}}$ 防止过度破坏最优个体, 加快 收玫, 此时的交叉率 $P_{\mathrm{c}}$ 和变异率 $P_{\mathrm{m}}$ 并不为 0 , 不会 陷入局部最优解。

5) 迭代终止条件。本算法的终止条件为: 当算 法达到最大迭代次数时终止, 本算法的最大迭代次数 为 200; 或者当子代的群体平均适应度与上一代群体 的平均适应度的差小于 0.001 同时子代个体中的最大 适应度不等于 0 时算法终止。由于实验得到的图像经 过预处理后, 图像中大部分的区域都是属于低灰度 级, 为了防止算法在初始几代中出现连续两代群体的 适应度值全部为 0 而导致终止, 故采取当子代个体中 最大适应度不等于 0 时方可终止迭代。

本文的图像分割算法流程图如图 2 所示。

\section{3 实验与分析}

\section{1 试件的渗透检测}

本文实验利用超声红外热像技术检测奥氏体不 锈钢焊缝裂纹, 焊㖓裂纹是通过焊接工艺在焊缝上随 机产生的表面裂纹。为了验证后续实验的正确性, 首 先对实验试件进行渗透检测, 以确定裂纹的数量及位 置, 渗透检测结果如图 3 所示。

\section{2 图像预处理}

本研究选取在超声波激励幅值 $A=32 \mu \mathrm{m}$ 、激励频 率 $f=20 \mathrm{kHz}$ 、激励时间 $t=2 \mathrm{~s}$ 实验条件下的热波序列 图为例进行图像处理。该实验的红外热波序列图如图 4 所示。由于奥氏体不锈钢试件反射率高、采集设备、 操作等原因, 导致采集到的实验图像具有一定背景噪 声。本文首先利用多图像平均法对序列图处理得到平 均图像, 再将平均图像减去初始图像去背景噪声。 


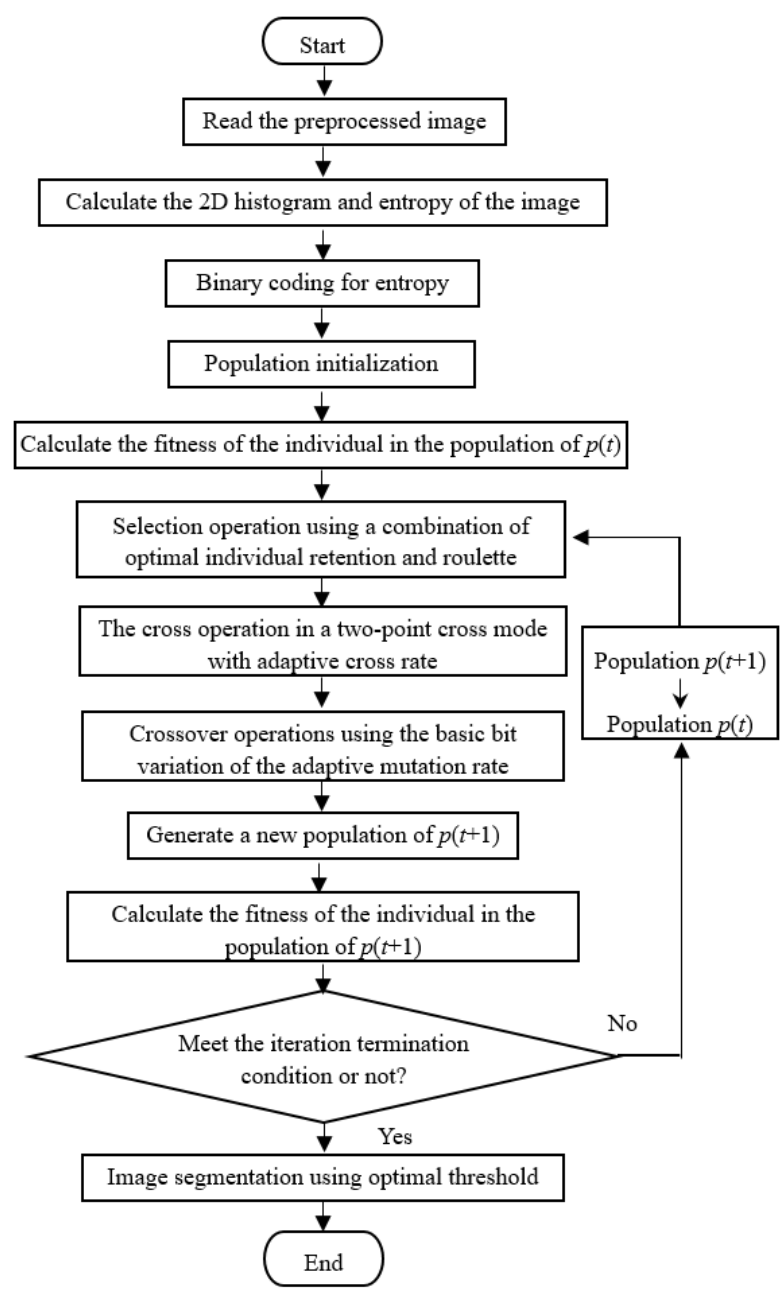

图 2 图像分割流程图

Fig.2 Image segmentation flow chart

在差分运算后得到的图像中, 一些比较明显的、 具有干扰性的背景噪声被较好地抑制, 但是仍然存在

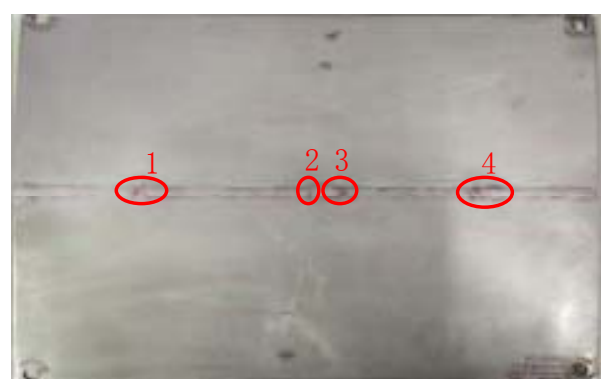

(a) 实验试件 (a) Specimen

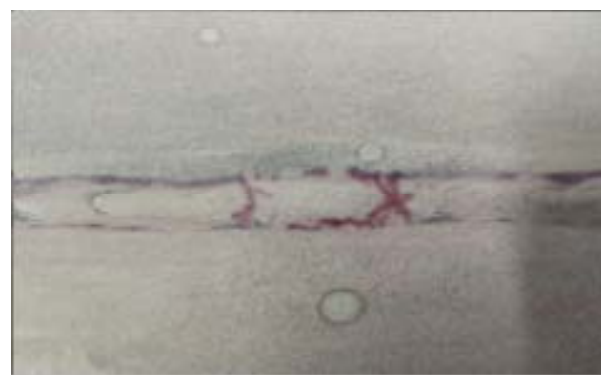

(c) 2 号和 3 号裂纹 (c) Crack No.2 and No.3

图 3 渗透检测结果图
着一些由试件高频振动引起的细微干扰信号, 为此, 本文采用形态学开运算去除细微的干扰信号。图像预 处理过程如图 5 所示。

\section{3 图像分割}

对预处理后的图像进行分割, 利用本文的分割方 法进行分割的结果如图 6 所示。

从图中可以看出, 尽管由于热的传播使得裂纹区 域面积比实际情况稍大, 但还是能够准确地分割出裂 纹区域。从图 3 的渗透检测实验结果可以看出, 2 号 裂纹和 3 号裂纹两个裂纹之间的间隔非常短, 热流的 传导使得两个裂纹摩擦生热产生的温度场相互影响 形成一个升温区域, 故图 6 中的 2 号裂纹和 3 号裂纹 融合成同一块目标区域了。

为验证本文算法的准确性, 本文利用穷举法和简 单遗传算法对同一张图像进行分割, 分割的结果如图 7、图 8 所示。对比图 6 可以看出, 相比于简单遗传算 法, 改进的自适应遗传算法分割的结果更接近于穷举 法的分割结果, 表明了本文算法具有更高的分割精度。

进一步地, 本文对该算法的分割速度进行验证, 由于遗传算法的分割具有随机性, 每次的分割结果和 效率都会有细微的差别。因此, 本文利用每种算法对 同一张图像进行 20 次分割, 取其平均值, 实验数据 如表 1 所示。

从表 1 中可以看出, 基于改进遗传算法的二维最大 熵分割速度远远高于穷举法的分割速度, 分割时间从 $54.227 \mathrm{~s}$ 降低到 $0.4115 \mathrm{~s}$; 相比简单遗传算法, 改进遗传 算法的平均运算时间从 $0.6045 \mathrm{~s}$ 缩短到了 $0.4115 \mathrm{~s}$, 降 低了 $31.93 \%$, 分割速度也有了明显的改善。

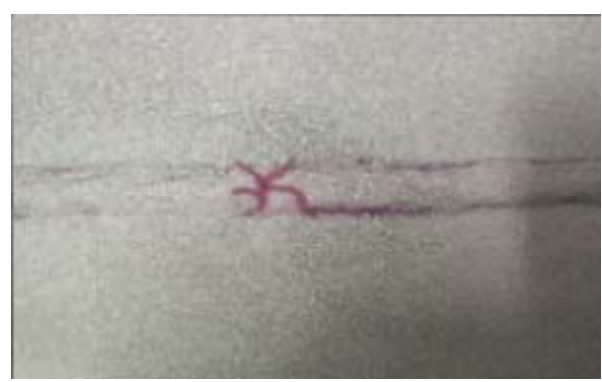

(b) 1 号裂纹 (b) Crack No.1

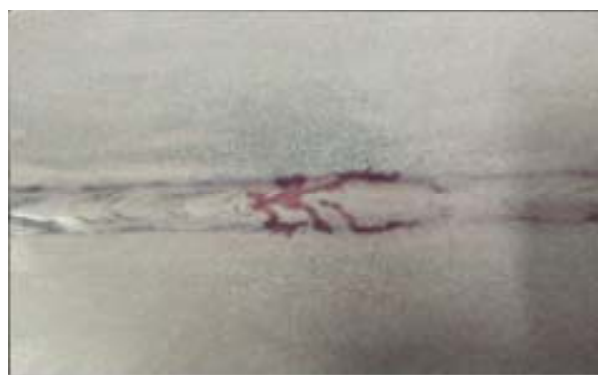

(d) 4 号裂纹 (d) Crack No.4

Fig.3 Penetration test results 


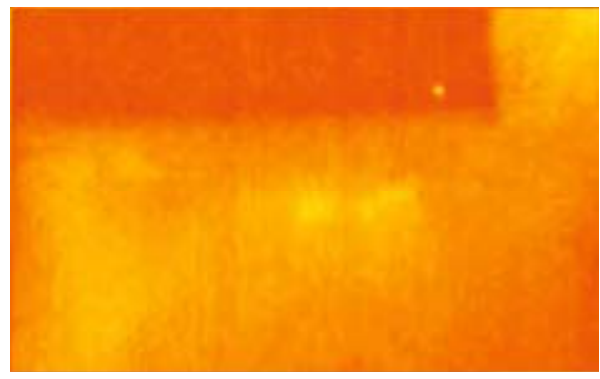

(a) $t=0 \mathrm{~s}$

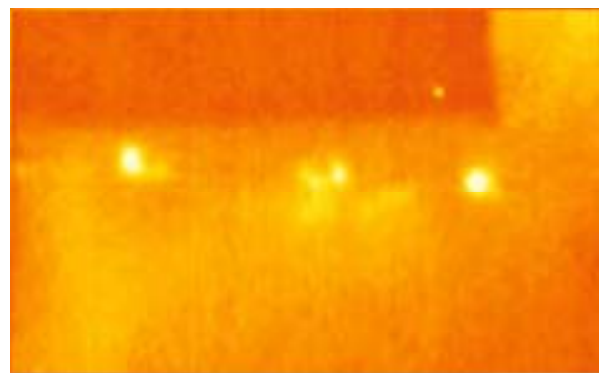

(c) $t=2 \mathrm{~s}$

图 4 试件表面红外热波序列图（ $A=32 \mu \mathrm{m})$

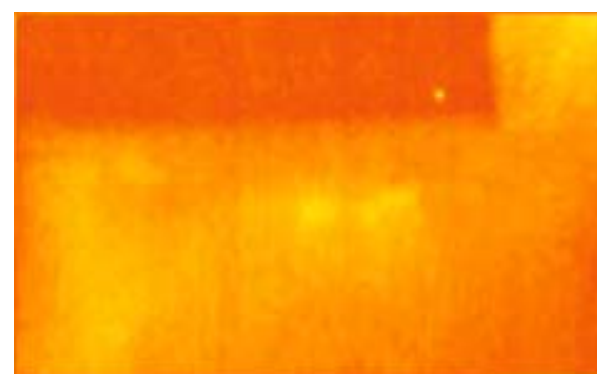

(a) 初始图像 (a) Original image

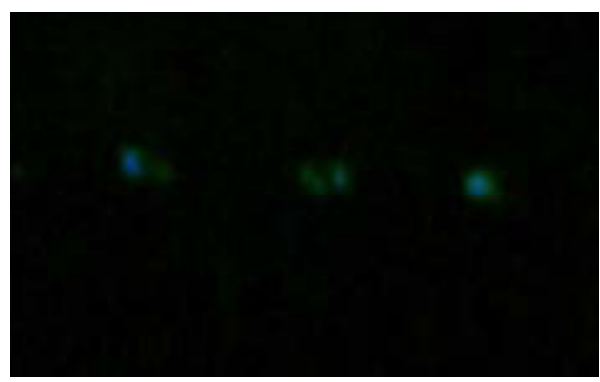

(c) 图像差分结果 (c) Difference operation result 图 5 图像预处理

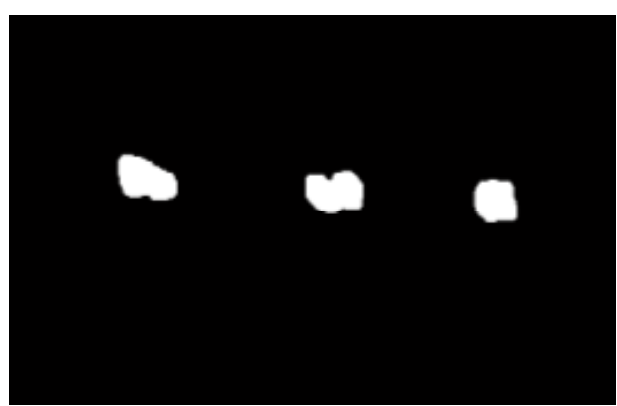

图 6 改进遗传算法分割结果

Fig.6 Segmentation result using improved genetic method

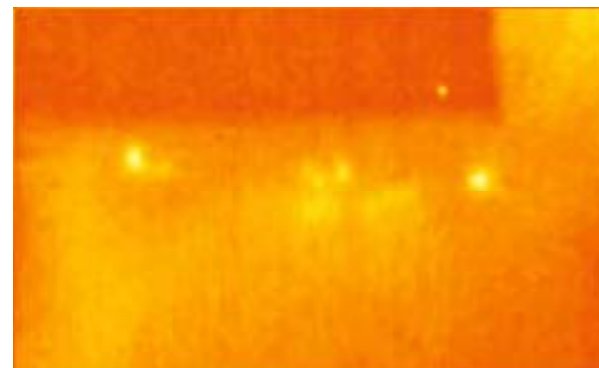

(b) $t=1 \mathrm{~s}$

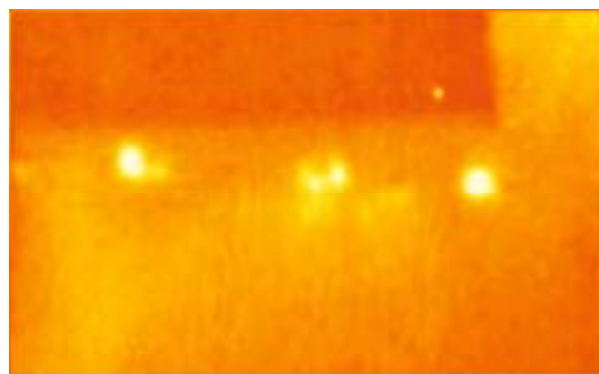

(d) $t=3 \mathrm{~s}$

Fig.4 Infrared heat wave sequence of specimen surface $(A=32 \mu \mathrm{m})$

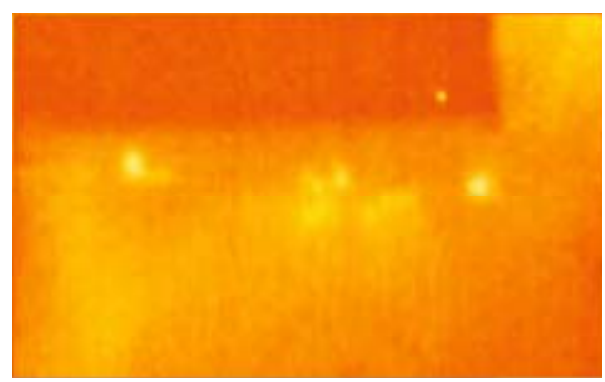

(b) 平均图像 (b) Averaged image

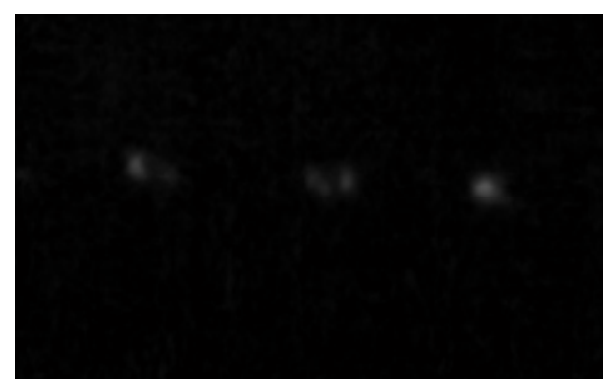

(d) 图像开运算结果 (d) Open operation result

Fig.5 Image preprocessing

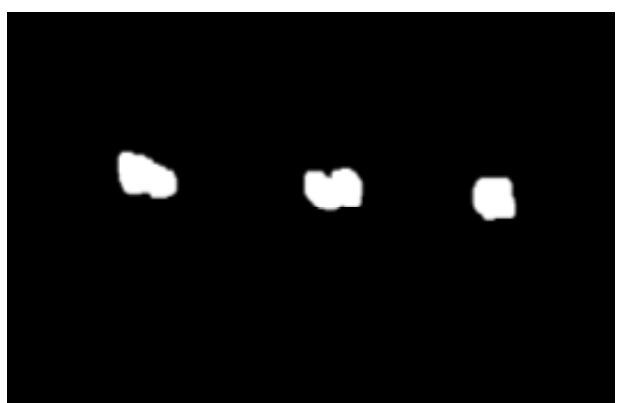

图 7 穷举法分割结果

Fig.7 Segmentation result using exhaustive method 


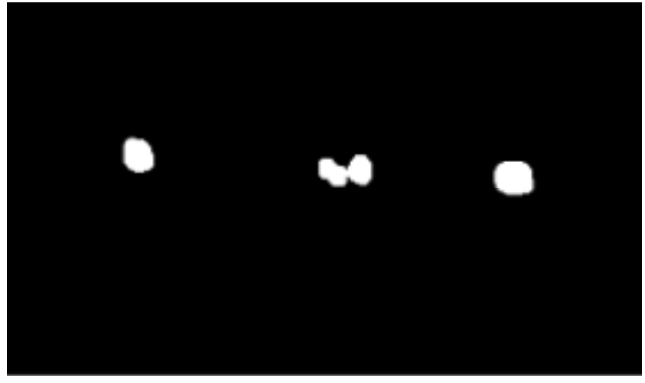

图 8 基本遗传算法分割结果

Fig. 8 Segmentation result using basic genetic algorithm

为了进一步说明本文算法的有效性, 本文对在 另外两个激励幅值 $A=24 \mu \mathrm{m} 、 A=28 \mu \mathrm{m}$ 激励下的

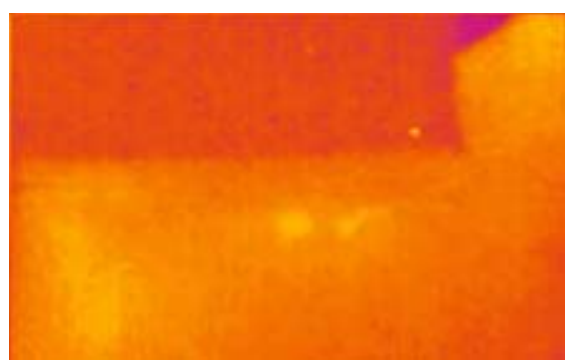

(a) 初始图像 (a) Original image

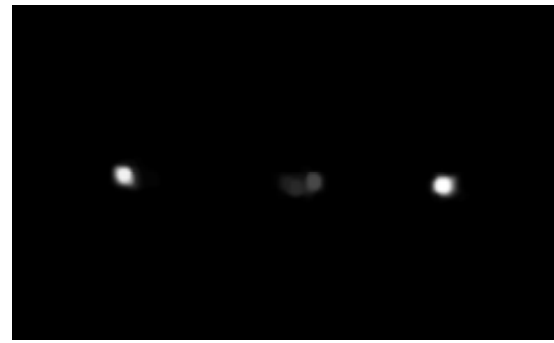

(c) 预处理图像 (c) Preprocessed image

图 9 图像分割结果 $(A=24 \mu \mathrm{m})$

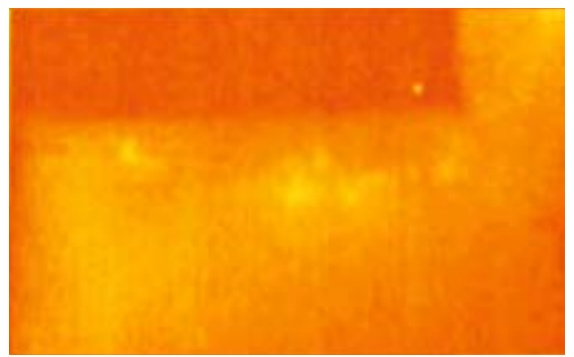

(a) 初始图像 (a) Original image

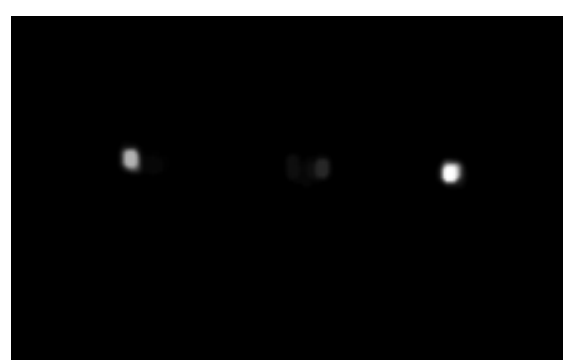

(c) 预处理图像 (c) Preprocessed image 图 10 图像分割结果 $(A=28 \mu \mathrm{m})$
红外热波序列图进行图像处理, 激励频率 $f=20$ $\mathrm{kHz}$ 、激励时间 $t=2 \mathrm{~s}$, 利用本文的算法的分割过程 及结果如图 9 和图 10 所示。

表 1 不同算法的图像分割速度比较

Table 1 Segmentation speed of different methods

\begin{tabular}{cc}
\hline Method & Processing time/s \\
\hline Exhaustive method & 54.227 \\
2D maximum entropy segmentation & 0.6045 \\
based on simple genetic algorithm & \\
$\begin{array}{c}\text { 2D maximum entropy segmentation } \\
\text { based on improved genetic } \\
\text { algorithm }\end{array}$ & 0.4115
\end{tabular}

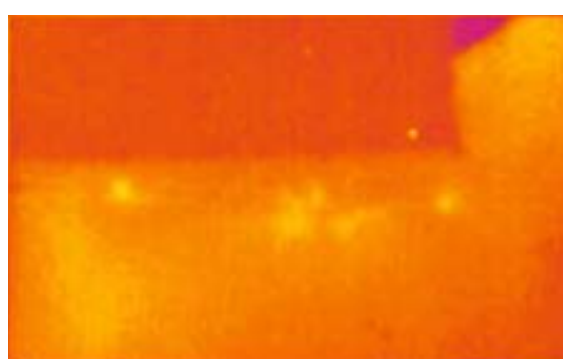

(b) 平均图像 (b) Averaged image

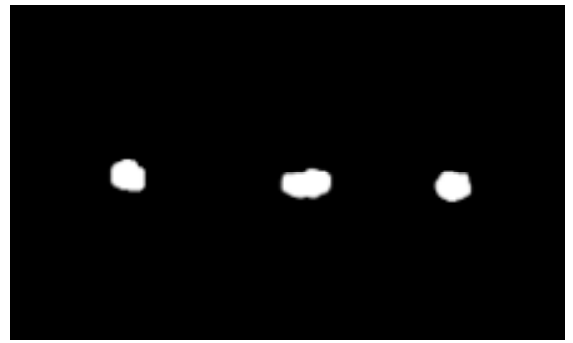

(d) 图像分割 (d) Segmentation result

Fig.9 Segmentation result $(A=24 \mu \mathrm{m})$

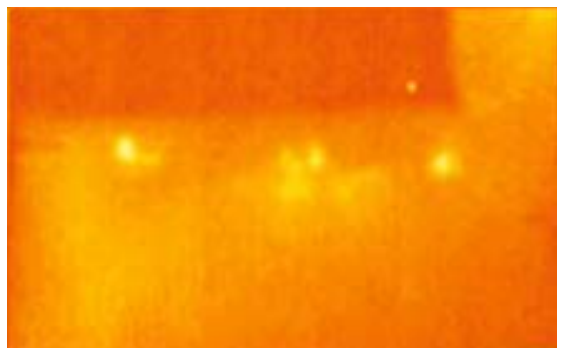

(b) 平均图像 (b) Averaged image

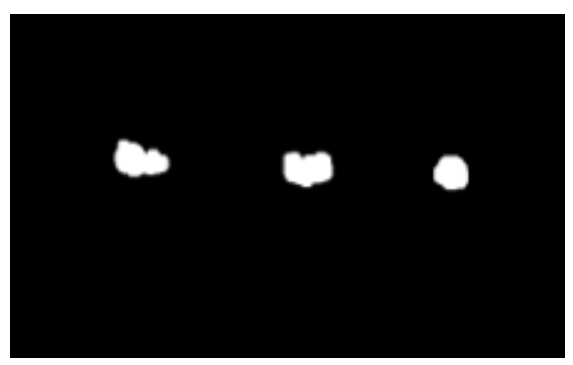

(d) 图像分割 (d) Segmentation result

Fig.10 Segmentation result $(A=28 \mu \mathrm{m})$ 
从图中可以看出, 在两个激励幅值 $A=24 \mu \mathrm{m} 、 A$ $=28 \mu \mathrm{m}$ 激励下的红外热波序列图都可以利用本文的 算法进行图像分割, 且都能较好地实现对裂纹缺陷区 域的提取，完成缺陷区域和背景区域的区分。

\section{4 缺陷定位}

在图像分割的基础上, 本文进一步采用连通区域 标记提取出缺陷的位置。本文采用 8 邻接方式进行连 通区域标记, 提取连通区域的个数。然后再求得各个 连通区域的质心, 利用质心来确定裂纹区域的大致位 置。本文对阈值分割后的二值图像进行连通区域标 记, 如图 11 所示。将利用连通区域标记提取的缺陷 位置与渗透检测得到的缺陷位置进行对比, 如表 2 所 示。表中的数值表示缺陷距试件左边缘的水平距离。

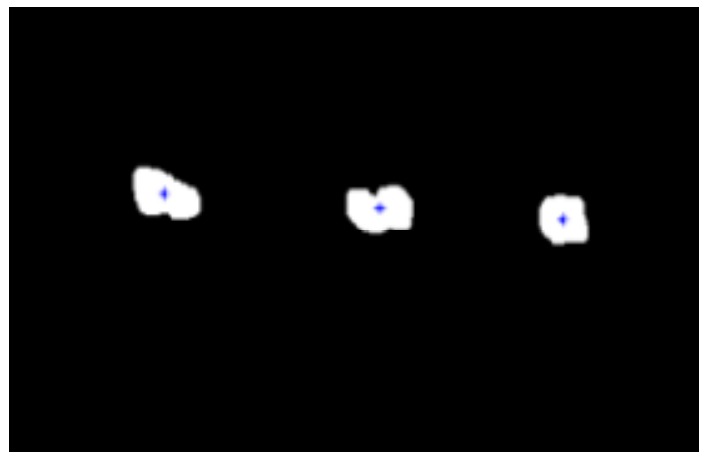

图 11 连通区域标记图

Fig.11 Connected area with marker

表 2 不同方法下的裂纹位置对比

Table 2 Position of the crack obtained from different method

\begin{tabular}{ccc}
\hline Crack & Penetrant testing & Connected area marker \\
\hline No.1 & $55-75 \mathrm{~mm}$ & $67 \mathrm{~mm}$ \\
No.2 & $145 \mathrm{~mm}$ & $160 \mathrm{~mm}$ \\
No.3 & $155-168 \mathrm{~mm}$ & \\
No.4 & $225-245 \mathrm{~mm}$ & $240 \mathrm{~mm}$ \\
\hline
\end{tabular}

从表 2 中可以看出, 利用连通区域标记提取的裂 纹缺陷位置信息与本文的渗透检测实验结果的裂纹 位置信息基本一致, 表明了本章提出的红外图像处理 方法能够较好地对超声红外实验结果进行处理, 实现 缺陷的识别与定位, 提高超声红外检测方法的缺陷识 别率。

\section{4 结论}

本文对奥氏体不锈钢焊缝裂纹在超声激励作用下 的红外热波序列图进行图像处理, 首先利用多图像平 均法对红外热波系列图进行处理, 将得到的平均图像 与序列图中的第一帧初始图像进行图像差分运算去背 景, 然后用形态学开运算去除由实验试件高频振动引
起的图像漂移而产生的干扰噪声。在此基础上, 提出 一种结合改进的自适应遗传算法和二维最大熵的图 像方法, 对预处理后的图像进行分割, 提取图像中 的裂纹区域。最后, 利用连通区域标记提取裂纹区 域的位置信息。实验结果表明: 利用图像差分运算 和形态学开运算相结合的预处理能对超声红外热波 序列图较好地去噪; 结合改进的自适应遗传算法和 二维最大熵的图像分割方法能对图像实现较好地分 割, 相比于穷举法, 它的分割速度有了很大的提高, 分割时间从 $54.227 \mathrm{~s}$ 降低到 $0.4115 \mathrm{~s}$, 相比于简单遗传 算法, 它的分割精度更高, 同时分割速度平均提高了 $31.93 \%$; 利用连通区域标记能对缺陷实现准确的定位。

\section{参考文献：}

[1] 闵庆旭, 冯辅周, 徐超,等. 超声红外锁相热像技术用于金属平板疲劳 裂纹的检测 [J]. 红外技术, 2018, 40(1): 95-98.

MIN Qingxu, FENG Fuzhou, XU Chao, et al. Detection of fatigue cracks in metal plates using lock-in vibro thermography[J]. Infrared Technology, 2018, 40(1): 95-98

[2] 冯辅周, 张超省, 江鹏程, 等. 超声红外热像技术中缺陷的自动识别 [J]. 激光与红外, 2012, 42(10): 1149-1153.

FENG Fuzhou, ZHANG Chaosheng, JIANG Pengcheng, et al. Automatic identification of crack in ultrasonic infrared imaging technology[J]. Laser \& Infrared, 2012, 42(10): 1149-1153.

[3] 郑凯, 江海军, 陈力. 红外热波无损检测技术的研究现状与进展 [J]. 红外技术, 2018, 40(5): 401-411.

ZHENG Kai, JIANG Haijun, CHEN Li. Infrared thermography NDT and its development[J]. Infrared Technology, 2018, 40(5): 401-411.

[4] 刘慧. 超声红外锁相热像无损检测技术的研究 [D]. 哈尔滨: 哈尔滨工 业大学, 2010 .

LIU Hui. Research on Ultrasound Infrared Lock-in Thermography for Non-destructive Testing[D]. Harbin: Harbin Institute of Technology, 2010.

[4] 秦雷, 刘俊岩, 龚金龙, 等. 超声红外锁相热像技术检测金属板材表 面裂纹[J]. 红外与激光工程, 2013(5): 1123-1130.

QIN Lei, LIU Junyan, GONG Jinlong, et al. Testing surface crack defects of sheet metal with ultrasonic lock-in thermography[J]. Infrared and Laser Engineering, 2013(5): 1123-1130.

[6] 郑凯, 张淑仪, 蔡士杰. 超声激发下缺陷红外信号的识别[J]. 无损检 测, 2008, 30(10):96-98.

ZHENG Kai, ZHANG ShuYi, CAI Shijie. Defect recognition in ultrasonic infrared thermography[J]. Nondestructive Testing, 2008, 30(10): 96-98.

[7] 李俊山, 杨威, 张雄美. 红外图像处理、分析与融合 $[\mathrm{M}]$. 北京: 科学出 版社, 2009. 
LI Junshan, YANG Wei, ZHANG Xiongmei. Infrared Image Processing, Analysis and Fusion[M]. Beijing: Science Press, 2009.

[8] 杜晓晨, 刘建平. 基于二维 OTSU 和遗传算法的红外图像分割方法 [J]. 红外技术, 2005, 27(1): 66-69.

DU Xiaochen, LIU Jianping. Infrared image segmentation method based on 2D maximum between-cluster variance and genetic algorithm[J]. Infrared Technology, 2005, 27(1): 66-69.

[9] Holland John H. Genetic algorithms and the optimal allocation of trials[J]. SIAM Journal on Computing, 1973, 2(2): 88-105.

[10] 李茂民. 一种基于改进遗传算法的图像分割研究及应用 [D]. 赣州: 江西理工大学, 2009.

LI Maoming. Research and Application of an Improved Genetic Algorithm for Image Segmentation[D]. Ganzhou: Jiangxi University of
Science and Technology, 2009.

[11] 李宏言, 盛利元, 陈良款, 等. 基于二维最大熵原理和改进遗传算法 的图像國值分割 [J]. 计算机与现代化, 2007(2): 34-38.

LI Hongyan, SHENG Liyuan, CHEN Liangkuan, et al. Image thresholding segmentation based on 2D maximum entropy principle and improved genetic algorithm[J]. Computer and Modernization, 2007(2): 34-38.

[12] Antoniol G, Ceccarelli M. Microarray image gridding with stochastic search based approaches[J]. Image and Vision Computing, 2007, 25(2): $155-163$.

[13] 玄光男. 遗传算法与工程设计[M]. 北京: 科学出版社, 2000. XUAN Guangnan. Genetic Algorithm and Engineering Design[M]. Beijing: Science Press, 2000.

\section{《红外技术》第八届编辑委员会名单}

荣誉主编：褚君浩 姜会林 曾 毅 曾桂林

主 编: 苏君红

执行主编：姚立斌

副主编：刘逸平唐 剑李凯朱颖峰陈钱 蔡 教 何力金伟其 郑云峰 (常 务)

编委 (以姓氏笔画为序)

马文坡王双保王霞石峰卢进史衍丽白廷柱白瑜 朱颖峰 刘逸平 麦绿波苏君红巫广华李云红李凯李晓峰

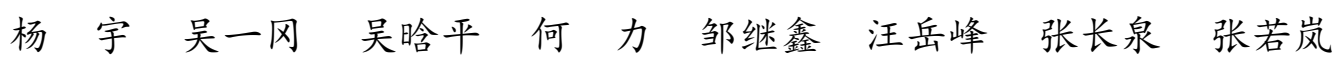
张建奇陆卫陈钱陈效双陈福胜金伟其郑云峰郑凯 赵勋杰 赵 俊胡伟达 施加林姚立斌顾国华 凌福日 郭 杰 唐利斌唐良瑞唐 剑姬㭉斌常本康 葛宝瑧蒋亚东 曾 宇 蔡 毅 熊 平 